\title{
Mild White Matter Changes in Un-medicated Obsessive-Compulsive Disorder Patients and Their Unaffected Siblings
}

\author{
Siyan Fan 1,2,3, Odile A. van den Heuvel 1, 2, 4,5*, Danielle C. Cath ${ }^{3,6}$, \\ Ysbrand D. van der Werf ${ }^{1,4,7}$, Stella J. de Wit ${ }^{1,2,4,5}$, Froukje E. de Vries ${ }^{1,2,4}$, \\ Dick J. Veltman ${ }^{2,4}$ and Petra J. W. Pouwels ${ }^{4,8}$ \\ ${ }^{1}$ Department of Anatomy and Neurosciences, VU University Medical Center, Amsterdam, Netherlands, ${ }^{2}$ Department of \\ Psychiatry, VU University Medical Center, Amsterdam, Netherlands, ${ }^{3}$ Department of Social and Behavioural Science, Utrecht \\ University, Utrecht, Netherlands, ${ }^{4}$ Neuroscience Campus Amsterdam, VU/VU University Medical Center, Amsterdam, \\ Netherlands, ${ }^{5}$ The OCD Team, Haukeland University Hospital, Bergen, Norway, ${ }^{6}$ Academic Anxiety Center Altrecht, Utrecht, \\ Netherlands, ${ }^{7}$ Netherlands Institute for Neuroscience, Amsterdam, Netherlands, ${ }^{8}$ Department of Physics and Medical \\ Technology, VU University Medical Center, Amsterdam, Netherlands
}

OPEN ACCESS

Edited by:

Kirsten R. Müller-Vahl,

Medical Doctor, Germany

Reviewed by:

Kerstin Jessica Plessen,

University of Copenhagen, Denmark

Tino Prell,

University Hospital Jena, Germany

*Correspondence:

Odile A. van den Heuvel oa.vandenheuvel@vumc.n

Specialty section: This article was submitted to Child and Neurodevelopmental

Psychiatry,

a section of the journal

Frontiers in Neuroscience

Received: 28 September 2015 Accepted: 14 December 2015

Published: 11 January 2016

Citation:

Fan S, van den Heuvel OA, Cath DC, van der Werf YD, de Wit SJ, de

Vries FE, Veltman DJ and Pouwels PJW (2016) Mild White Matter Changes in Un-medicated Obsessive-Compulsive Disorder

Patients and Their Unaffected Siblings. Front. Neurosci. 9:495. doi: 10.3389/fnins.2015.00495
Objective: Obsessive-compulsive disorder (OCD) is a common neuropsychiatric disorder with moderate genetic influences and white matter abnormalities in frontal-striatal and limbic regions. Inconsistencies in reported white matter results from diffusion tensor imaging (DTI) studies can be explained, at least partly, by medication use and between-group differences in disease profile and stage. We used a family design aiming to establish whether white matter abnormalities, if present in un-medicated OCD patients, also exist in their unaffected siblings.

Method: Forty-four OCD patients, un-medicated for at least the past 4 weeks, 15 of their unaffected siblings, and 37 healthy controls $(\mathrm{HC})$ underwent DTI using a 3-Tesla MRI-scanner. Data analysis was done using tract-based spatial statistics (TBSS). Fractional anisotropy (FA), axial diffusivity (AD), radial diffusivity (RD), and mean diffusivity (MD) values were compared within seven skeletonised regions of interest (ROls), i.e., corpus callosum, bilateral cingulum bundle, bilateral inferior longitudinal fasciculus/ frontal-occipital fasciculus (ILF/FOF) and bilateral superior longitudinal fasciculus (SLF).

Results: Un-medicated OCD patients, compared with $\mathrm{HC}$, had significantly lower FA in the left cingulum bundle. FA was trend-significantly lower in all other ROls, except for the corpus callosum. Significant three-group differences in FA (and in RD at trend-significant level) were observed in the left cingulum bundle, with the unaffected siblings representing an intermediate group between OCD patients and $\mathrm{HC}$.

Conclusions: OCD patients showed lower FA in the left cingulum bundle, partly driven by trend-significantly higher values in $\mathrm{RD}$. Since the unaffected siblings were found to be an intermediate group between OCD patients and $\mathrm{HC}$, this white matter alteration may be considered an endophenotype for OCD.

Keywords: diffusion tensor imaging, fractional anisotropy, obsessive-compulsive disorder, endophenotype 


\section{INTRODUCTION}

Obsessive-compulsive disorder (OCD) is a debilitating neuropsychiatric disorder characterized by obsessions (intrusive recurrent thoughts) and/or compulsions (repetitive behaviors). OCD is moderately heritable (Hudziak et al., 2004; Van Grootheest et al., 2007), with heritability rates between 40 and $45 \%$ (Hudziak et al., 2004) and first degree relatives of the patients having a 4-10 times higher risk of developing OCD (Nestadt et al., 2000), depending on age of the proband. At the same time, the genetic basis of OCD is complex, multi-factorial, and under strong environmental influence (Grisham et al., 2008). Both structural and functional neural correlates of OCD have been found in the unaffected first-degree relatives of OCD patients (Chamberlain et al., 2008; Menzies et al., 2008b; de Wit et al., 2012; de Vries et al., 2014), suggesting that at least in part, the alterations are state-independent and might be regarded as correlates of genetic vulnerability, also called endophenotypes (Gottesman and Gould, 2003).

In contrast to the large literature on gray matter alterations in OCD (Radua and Mataix-Cols, 2009; Rotge et al., 2009; de Wit et al., 2014), white matter abnormalities in OCD have caught relatively less attention. A recent OCD Brain Imaging Consortium mega-analysis (de Wit et al., 2014), involving 412 OCD patients and 368 healthy controls (HC), reported decreased white matter volumes in frontal regions in the patient group, suggesting abnormalities of white matter connections between the prefrontal and subcortical regions within the frontal-striatal circuits. These results were consistent with some previous studies on white matter volume (van den Heuvel et al., 2009; Togao et al., 2010). White matter volume alterations have also been reported for the parietal and occipital lobes (Riffkin et al., 2005; Lázaro et al., 2009, 2011).

Although potentially related to white matter volume, the microstructure of the white matter tracts may provide additional insights into the pathophysiology of OCD. Diffusion tensor imaging (DTI) is a widely used neuroimaging technique to study brain tissue microstructure by quantification of the diffusion characteristics of water molecules (Le Bihan et al., 2001). Anisotropy, generally expressed as fractional anisotropy (FA), is a directionally dependent property of water diffusivity. FA in white matter reflects the underlying characteristics of microstructure, such as fiber density, axonal diameter, thickness of the myelin sheaths, and directionality of the fibers (Koch et al., 2014). FA is derived from the three eigenvalues of the diffusion tensor: $\lambda 1, \lambda 2$, and $\lambda 3$. The largest eigenvalue $(\lambda 1)$, or axial diffusivity (AD), has been found to be a possible marker for axonal injury. The average of the two smaller eigenvalues $\lambda 2$ and $\lambda 3$, or radial diffusivity (RD) has been suggested as an indicator of myelin damage (Song et al., 2003; Fan et al., 2012). Mean diffusivity (MD) is the average of all three eigenvalues.

A recent DTI meta-analysis by Radua et al., comparing 204 OCD patients with 231 matched $\mathrm{HC}$, showed reduced FA in the corpus callosum, the cingulum bundle, the inferior longitudinal fasciculi (ILF)/the frontal-occipital fasciculi (FOF), and the superior longitudinal fasciculi (SLF). Whether these alterations reflect state or trait effects (i.e., are the result of adaptive changes as a consequence of disease) or underlie disease vulnerability (and thus can be considered an endophenotype of OCD), cannot be deduced from the classical case-control studies. Therefore, extension to a family-based approach is warranted.

Menzies et al. (2008b) were the first to show that both OCD patients and their first-degree relatives had reduced FA in the right inferior parietal white matter and increased FA in the right medial frontal region. However, their results may have been partly confounded by suboptimal matching between the groups and medication use. Also, they did not explore differences in diffusivity parameters underlying the FA alterations. A combined DTI/voxel-based morphometry study using a monozygotic (MZ) twin design reported white matter characteristics of $M Z$ twins who were concordant and discordant on obsessive-compulsive traits (den Braber et al., 2011). This study reported effects for environmental as opposed to genetic influences on regional white matter volume: the predominant FA decrease found in inferior frontal regions in the MZ concordant-high vs. concordant-low twins was suggested to reflect genetic (trait) effects, whereas the within MZ discordant twin comparison revealed increased dorsolateral prefrontal white matter changes in the high scoring twins, thought to reflect changes due to environmental effects. More recently, Shaw et al. investigated adult and adolescent OCD patients and their unaffected siblings and found similar morphological abnormalities in cortical and subcortical regions of caudate nucleus, thalamus and the right orbitofrontal cortex. Besides, both OCD patients and unaffected siblings, as compared with healthy controls, showed increased thickness of the right precuneus (Shaw et al., 2015).

In summary, available evidence for white matter abnormalities in OCD is largely inconsistent. We suggest several possible reasons for the reported discrepancies: (1) in OCD, white matter alterations might be subtle thus difficult to detect; (2) medication effects seem to confound the findings (Yoo et al., 2007; Fan et al., 2012; Benedetti et al., 2013; Radua et al., 2014); (3) results are highly variable across pediatric, adolescent and adult patients due to changes in white matter architecture throughout brain development (Peters et al., 2012); (4) debate has risen recently on whether FA alone is sufficient and sufficiently representative to indicate changes in white matter microstructure (Hasan, 2006; Fan et al., 2012; Szczepankiewicz et al., 2014); and (5) small samples and inconsistent methodologies have been used (Radua et al., 2014).

In this study, we aimed to replicate and extend previous findings, by exploring white matter microstructure in a group of un-medicated adult OCD patients, their unaffected siblings and gender and age-matched HC. By using tract-based spatial statistics (TBSS), we first aimed to investigate whether there were any abnormalities of FA in OCD patients compared with HC, and to investigate whether these between-group differences could be explained by any of the diffusivity measures (i.e., AD and $\mathrm{RD})$. Finally, we aimed to explore whether alterations in diffusion parameters also existed in the unaffected siblings, to disentangle whether white matter changes are cause or consequence of the disease.

We used a region-of-interest (ROI) approach by selecting 7 ROIs, based on the results from the meta-analysis of Radua 
et al. (2014), corpus callosum, the bilateral cingulum bundle, the bilateral ILF/FOF and the bilateral SLF. We hypothesized that OCD patients compared with HC would show lower FA with changes in diffusivity values in the ROIs. Moreover, based on the familiality of OCD we expected that these abnormalities would be shared (as a trait), at least partly, in the unaffected siblings of the patients.

\section{METHODS AND MATERIALS}

\section{Participants}

Forty-four OCD patients who were un-medicated for at least 4 weeks when participating in the study (mean age 38.5 year, SD $=9.9$ ), 15 of their unaffected siblings (mean age 38.1 year, $\mathrm{SD}=14.1$ ), and $37 \mathrm{HC}$ (mean age 39.5 year, $\mathrm{SD}=11.5$ ) were included. The groups were matched on age, gender, handedness, and education level. OCD patients were recruited from the outpatient clinics within the Netherlands OCD Association cohort (Schuurmans et al., 2012), the Academic Anxiety Center Altrecht (Utrecht, the Netherlands), and by online advertisements. HC were recruited by local and online community advertisements.

All participants were screened on axis I psychiatric disorders using the Structured Clinical Interview for DSM-IV-TR Axis I Disorders (First et al., 2002). OCD symptom characteristics and severity were assessed with the Yale-Brown Obsessive Compulsive Scale (Y-BOCS, symptom list and severity scale) (Goodman et al., 1989) and the/Inventory-Revised (OCI-R) (Foa et al., 2002). Depressive symptoms were assessed with the Montgomery-Åsberg Depression Rating Scale (MADRS) (Montgomery and Asberg, 1979) and handedness with the Edinburgh Handedness Inventory (Oldfield, 1971).

Current psychoactive medication use, current or past psychosis, major physical illness, a history of a major neurological illness, and MRI contra-indications served as exclusion criteria. All the OCD patients were un-medicated for at least 4 weeks. No patients were excluded due to their psychiatric comorbidity (including tic disorder) and they could participate if they had a primary diagnosis of OCD without predominant hoarding. Siblings were included provided that they did not meet lifetime criteria for OCD and had no current DSM-IV-TR axis I diagnosis. Healthy control subjects had no current DSM-IV-TR axis I diagnosis and no family history of OCD. The local ethical review board of VU university medical center approved all procedures and all subjects provided written informed consent.

\section{MRI Acquisition}

MRI was performed using a whole-body 3-Tesla MR system (Signa HDxt, GE Healthcare, Milwaukee, USA) equipped with an eight-channel phased-array head coil. Diffusion weighted echoplanar imaging was collected in 30 diffusion weighted $(b=1000$ $\left.\mathrm{s} / \mathrm{mm}^{2}\right)$ and five reference $\left(b=0 \mathrm{~s} / \mathrm{mm}^{2}\right)$ volumes with 49 contiguous axial slices of $2.4 \mathrm{~mm}$ slice thickness for covering the whole brain (repetition time TR $14 \mathrm{~s}$, echo time TE $85 \mathrm{~ms}$ ). The acquired in-plane resolution was $2.0 \times 2.0 \mathrm{~mm}$, which was reconstructed to $1.0 \times 1.0 \mathrm{~mm}$. Parallel imaging was applied with an acceleration factor of 2 .

\section{Data Processing}

The diffusion MRI data were preprocessed for motion and eddy-current correction by using the FMRIB Software Library (FSL5; http://fsl.fmrib.ox.ac.uk/fsl). By fitting a tensor model to the raw diffusion data voxel-wise values of $F A, A D, R D$, and MD were obtained. All subjects' FA data were aligned to MNI standard space by using the non-linear image registration tool (FNIRT). Next, the mean FA image was created and thinned to create a mean FA skeleton, which represents the centers of all tracts common to the group. Each subject's aligned FA data was then projected onto this skeleton and the resulting data were fed into voxel-wise cross-subject statistics. The same non-linear registration was applied to the diffusivity parameters $A D, R D$ and MD.

\section{Statistical Analysis}

We compared the skeletonized diffusion parameters between OCD patients, the unaffected siblings and healthy controls using a ROI approach, including the 7 ROI's (i.e., the corpus callosum, the bilateral cingulum bundle, the bilateral ILF/FOF, and the bilateral SLF) based on the results from the meta-analysis of Radua et al. (2014). The Johns Hopkins University (JHU) ICBMDTI-81 white-matter labels atlas provided by FSL was chosen to define the ROIs. Permutation-based testing (5000 permutations) was carried out with Randomise, using Threshold-Free Cluster Enhancement (Smith and Nichols, 2009), and p-values were corrected for family wise error rate (FWE) taking into account multiple spatial comparisons. In addition to the ROI approach, for exploratory reasons, we performed whole-brain skeletonized voxel-wise statistics in order to check for group differences in the brain areas outside the ROIs. Corrected $p<0.05$ was considered significant, and uncorrected $p<0.05$ was considered a trend. Age and gender were added as covariates.

Randomise was used to compare FA values between OCD and HC. For each skeletonised ROI that had significantly different FA values between the two groups, we performed a threegroup comparison, now including the unaffected siblings, using Randomise. Of voxels that showed difference in the three-group comparison, the mean diffusion parameters (for FA, AD, RD, and $\mathrm{MD}$ ) were extracted for each subject (at uncorrected $p<$ 0.05). Subsequently, One-way ANOVA analysis was performed to investigate the overall group effect (three groups) of $\mathrm{AD}, \mathrm{RD}$, and MD $(p<0.05)$. Post-hoc 2 -sample-T tests were conducted to explore differences within groups. Within-group (OCD patients only) multiple regression analyses were carried out to investigate the relationship between FA and patients' disease severity (i.e., YBOCS severity scores).

\section{RESULTS}

\section{Demographic and Clinical Characteristics}

Age, sex, education level and handedness did not differ significantly between OCD patients, unaffected siblings, and HC (see Table 1). A main effect of group was found for Y-BOCS (symptom list and severity scale), the OCI-R and MADRS. OCD patients scored higher compared with $\mathrm{HC}$ and the unaffected 
siblings $(p<0.01)$; the sibling group did not significantly differ from the HC group on these measures. Twenty-five OCD patients (57\%) also met criteria for one or more comorbid current axis I diagnosis. No significant difference was found between OCD patients with $(n=25)$ or without $(n=19)$ comorbid diagnoses on demographic or other clinical measures $(p>0.16)$ (see Table 1).

\section{TBSS Analysis: FA Differences between OCD and HC}

OCD patients, compared with HC, had significantly lower FA in the left cingulum bundle (peak MNI coordinates $x=-24$, $y=-13, z=-32$; ROI-corrected $p<0.05$ ) as shown in Figure 1 and Table 2. At trend-significance level (uncorrected $p<0.05$ ) OCD patients showed a lower FA in a larger area of the left and in parts of the right cingulum bundle, bilateral ILF, and bilateral SLF (see Figures 2A,B and Table 2). No difference in FA was found in corpus callosum (uncorrected $p>0.14$ ). There were no areas in which OCD patients had higher FA than HC. TBSS analysis of the whole brain skeleton revealed no significant differences in FA between OCD and HC at the corrected level.

YBOCS and OCI-R scores were found neither positively nor negatively correlated with FA within the OCD patients group.

\section{Three Group}

\section{Comparisons-Endophenotype Analysis}

FA of the left cingulum bundle showed a significant effect of group (peak MNI coordinates $x=-28, y=-13, z=-31$; ROI-corrected $p<0.05$ ) as shown in Figure 1. Mean diffusion parameters values from the left cingulum bundle were extracted

TABLE 1 | Demographic and clinical characteristics of obsessive compulsive disorder patients, unaffected siblings, and healthy controls.

\begin{tabular}{|c|c|c|c|c|c|c|c|c|}
\hline & \multicolumn{2}{|c|}{ OCD Patients $(\mathrm{N}=44)$} & \multicolumn{2}{|c|}{ Siblings $(N=15)$} & \multicolumn{2}{|c|}{$\mathrm{HC}(\mathrm{N}=37)$} & \multirow{2}{*}{\multicolumn{2}{|c|}{$\begin{array}{c}\text { Analysis } \\
\mathrm{X}^{2}(\mathrm{df}=2) p\end{array}$}} \\
\hline & $N$ & $\%$ & $N$ & $\%$ & $N$ & $\%$ & & \\
\hline \multicolumn{9}{|l|}{ DEMOGRAPHIC MEASURES } \\
\hline Gender (men) & 22 & 50 & 11 & 73 & 18 & 49 & & 0.231 \\
\hline \multirow[t]{2}{*}{ Handedness (right) } & 37 & 84 & 12 & 80 & 32 & 86 & & 0.841 \\
\hline & Mean & SD & Mean & SD & Mean & SD & \multicolumn{2}{|c|}{$F(d f=2,93) p$} \\
\hline Age (years) & 38.5 & 9.9 & 38.1 & 14.1 & 39.5 & 11.5 & 0.1 & 0.893 \\
\hline Education levela & 5.9 & 1.9 & 6.0 & 1.5 & 5.8 & 1.9 & $0.1^{b}$ & 0.953 \\
\hline \multicolumn{9}{|l|}{ CLINICAL MEASURES } \\
\hline Y-BOCS severity (range 0-40) & 21.48 & 6.16 & 0.33 & 0.03 & 0 & 0 & $84.96^{b}$ & $<0.001$ \\
\hline \multicolumn{9}{|l|}{$\mathrm{OCl}-\mathrm{R}$} \\
\hline Total score & 23.75 & 12.27 & 3.27 & 3.35 & 3.22 & 4.80 & $59.23^{b}$ & $<0.001$ \\
\hline Washing score & 2.77 & 3.84 & 0.20 & 0.41 & 0.30 & 0.62 & $14.17^{b}$ & $<0.001$ \\
\hline Checking score & 6.25 & 3.85 & 0.47 & 0.74 & 0.49 & 0.93 & $52.66^{b}$ & $<0.001$ \\
\hline Order score & 4.75 & 3.85 & 0.87 & 1.41 & 0.81 & 1.53 & $31.07^{b}$ & $<0.001$ \\
\hline Obsession score & 5.36 & 3.83 & 0.53 & 0.92 & 0.32 & 1.36 & $50.20^{b}$ & $<0.001$ \\
\hline MADRS & 11.66 & 8.54 & 1.93 & 3.77 & 0.89 & 1.51 & $52.60^{b}$ & $<0.001$ \\
\hline
\end{tabular}

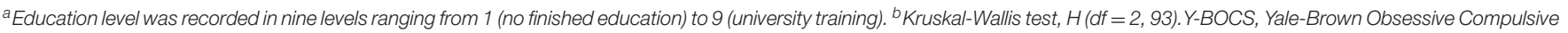
Scale; OCI-R, Obsessive-Compulsive Inventory-Revised; MADRS, Montgomery-Åsberg Depression Rating Scale; HC, Healthy Controls.

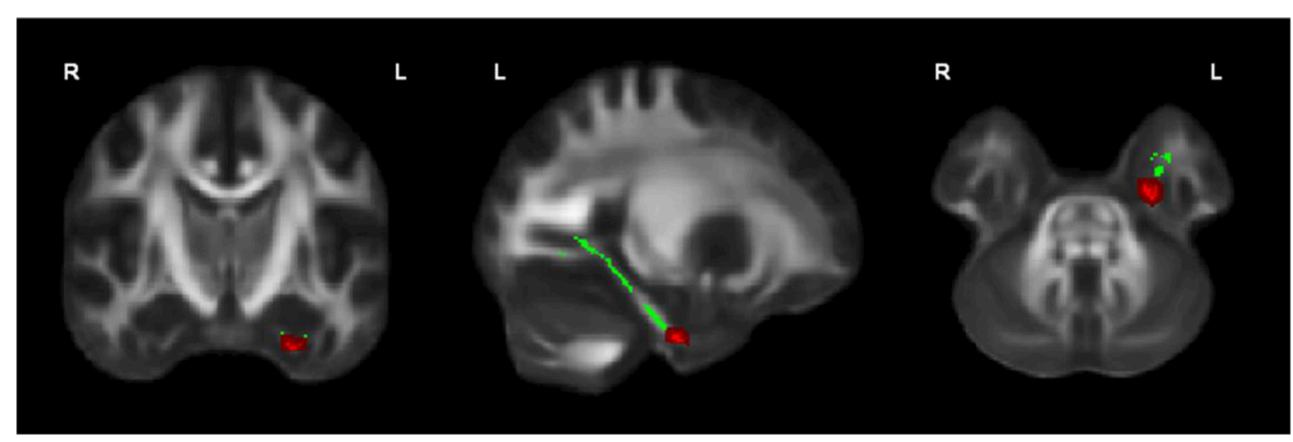

FIGURE 1 | OCD patients $(n=44)$ showed significantly lower FA than healthy controls $(n=37)$ in the left cingulum bundle, significant at $p<0.05$ (in red; corrected for multiple comparisons); for illustration purposes, the displayed skeletonized results were thickened. In green the parts of the skeleton within the pre-defined ROI. 
TABLE 2 | Locations of decreased FA in OCD patients compared to healthy controls.

\begin{tabular}{|c|c|c|c|c|c|}
\hline \multirow[t]{2}{*}{ ROls } & \multicolumn{3}{|c|}{ Peak coordinates } & \multirow[t]{2}{*}{ Cluster/Area } & \multirow{2}{*}{$\begin{array}{c}\text { OCD < HC } \\
p \text {-value } \\
\text { (uncorrected) }\end{array}$} \\
\hline & $x$ & $Y$ & $z$ & & \\
\hline $\begin{array}{l}\text { Left * cingulum } \\
\text { bundle }\end{array}$ & -24 & -13 & -32 & 38 & $0.001^{*}$ \\
\hline $\begin{array}{l}\text { Right cingulum } \\
\text { bundle }\end{array}$ & 25 & -5 & -33 & 28 & 0.003 \\
\hline Left ILF/FOF & -37 & -61 & 2 & 61 & 0.011 \\
\hline Right ILF/FOF & 43 & -50 & -17 & 110 & 0.003 \\
\hline Left SLF & -51 & -20 & 30 & 131 & 0.05 \\
\hline Right SLF & 56 & -12 & -22 & 105 & 0.03 \\
\hline
\end{tabular}

Peak MNI coordinates, cluster sizes and p-values (uncorrected). *indicates significance at $p<0.05$ corrected for multiple comparisons for ROI. FA, fractional anisotropy; IFO/FOF, inferior longitudinal fasciculus/frontal occipital fasciculus; SLF, superior longitudinal fasciculus; HC, healthy controls.

for all subjects based on voxels identified at uncorrected $p<0.05$ in the [FA (three-group comparison)] F-contrast. We selected values within this larger area of the left cingulum bundle (167 voxels), since the significant area at corrected $p<0.05$ is a relatively small cluster of 38 voxels located close to the inferior border of the cingulum bundle (see Figure 1). Figure 3 and Table 3 show that, after correction for multiple comparisons, mean FA was significantly lower in OCD patients compared with $\mathrm{HC}\left[F_{(2,93)}=6.40, p<0.001\right]$; mean $\mathrm{FA}$ values of unaffected siblings were intermediate between those of the OCD patients and of the HC, although post-hoc 2-sample-T comparisons did not show a significant difference between siblings and either the OCD patients or the $\mathrm{HC}$ ( $p=0.223$ and $p=1.000$ respectively).

Mean values of $\mathrm{AD}$ and $\mathrm{MD}$ in the left cingulum bundle (see Table 3) did not significantly differ across groups. RD values in this region showed a reverse pattern compared to FA values, with the OCD patients showing trend-significantly higher RD compared with $\mathrm{HC}$ and the unaffected siblings representing an intermediate group (see Table 3 and Figure 3). Although no effect of group was found for these diffusivity values after correction for multiple comparisons, a trend-significant effect of group was observed for the $\mathrm{RD}$ values $\left[F_{(2,93)}=3.34, p=0.04\right.$ uncorrected]. To test laterality of these results, we performed the same procedure for the diffusivity values in the right cingulum bundle, showing a similar pattern, although it does not surpass the trend-significant level.

\section{DISCUSSION}

The main finding of the present study is lower FA in the left cingulum bundle in un-medicated OCD patients compared with $\mathrm{HC}$, which partially replicates the findings from the meta-analysis conducted by Radua et al. (2014). We also found, in line with Radua et al., although at uncorrected significance level, lower FA in the other ROIs (except for the corpus callosum), i.e., the right cingulum bundle, the bilateral ILF/FOF, and the bilateral SLF.
Lower FA appeared to be associated with higher RD. The threegroup comparison showed that the unaffected siblings seemed to represent an intermediate group between the OCD patients and $\mathrm{HC}$ with respect to FA in the left cingulum bundle.

Lower FA in the cingulum bundle has been reported by most studies that investigated adult OCD patients (Koch et al., 2014; Radua et al., 2014). Increasing evidence from structural and functional neuroimaging research in recent years has emphasized the impact of deficits in temporo-parietal-occipital regions in the pathophysiology of OCD besides the frontal-striatal and fronto-limbic neurocircuitries (Menzies et al., 2008a; Piras et al., 2013). The cingulum bundle contains many short and long association fibers linking the frontal lobe with the temporal lobe (Schmahmann and Pandya, 2006), supporting communication between the prefrontal, parietal, and temporal regions (Jones et al., 2012). The finding of lower FA in the left cingulum bundle in OCD supports the suggested involvement of temporal and parietal regions in the pathophysiology of the disorder.

Although FA values in the right cingulum bundle, ILF/IFOF and SLF were only trend-wise lower in OCD patients compared with $\mathrm{HC}$, these findings may still be relevant since these differences were observed in all a-priori hypothesized regions bilaterally.

In general, the changes in diffusion parameters observed in these un-medicated OCD patients were subtle. Although Radua et al. (2014) in their meta-analyses reported widespread white matter abnormalities in OCD, no clear evidence so far has suggested OCD as a typical white matter disease (Koch et al., 2014). Furthermore, the lack of consistent DTI findings in the OCD literature may be explained, at least partly, by the impact of medication in OCD. Radua et al. (2014) reported that the lower FA was most prominent in samples with more medicated OCD patients. Benedetti et al. (2013) found that medicated OCD patients had higher RD in the corpus callosum and adjacent cingulate gyrus when compared to drug-naïve OCD patients and HC. Other studies in OCD also showed pharmacological treatment effects on white matter alterations (Yoo et al., 2007; Fan et al., 2012). Therefore, the subtle white matter abnormalities found in our un-medicated patient sample suggests that medication might be a potential confounder in most previous studies.

The here reported (trend-) significantly lower FA values all concern white matter regions that are spatially connected to temporal-parietal-occipital regions. Structural abnormalities found in both gray matter and white matter regions in posterior parts of the brain of OCD patients mainly concern the parietal lobe extending to the temporal and occipital lobes (Piras et al., 2013). These posterior regions are associated with cognitive functions including visuo-spatial functions, which have been consistently found to be impaired in OCD patients (Cohen et al., 1996; Savage et al., 1999). Our findings thus indirectly suggest abnormalities in these white matter microstructures might also be contributors to the cognitive impairments in visuo-spatial abilities.

The three-group comparison on FA in the left cingulum bundle showed a significant effect of group, with the unaffected siblings showing intermediate FA values. Mean $\mathrm{AD}$ and $\mathrm{MD}$ 
A

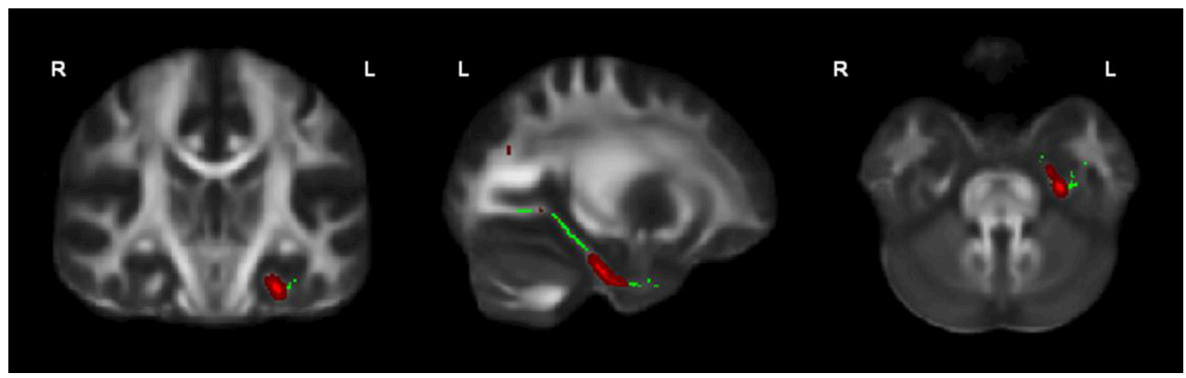

B

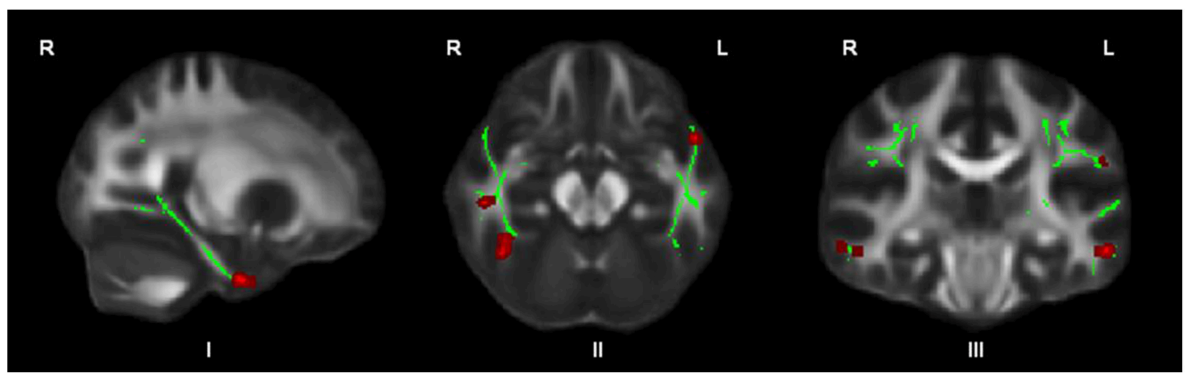

FIGURE 2 | OCD patients $(n=44)$ compared with healthy controls $(n=37)$ showed trend-significantly decreased FA in a larger part of the left cingulum bundle (A), in parts of the right cingulum bundle (I), bilateral ILF (II), and bilateral SLF (III) (in red; at $p \leq 0.05$ uncorrected) (B); for illustration purposes, the displayed skeletonized results were thickened. In green the parts of the skeleton within the pre-defined ROls.

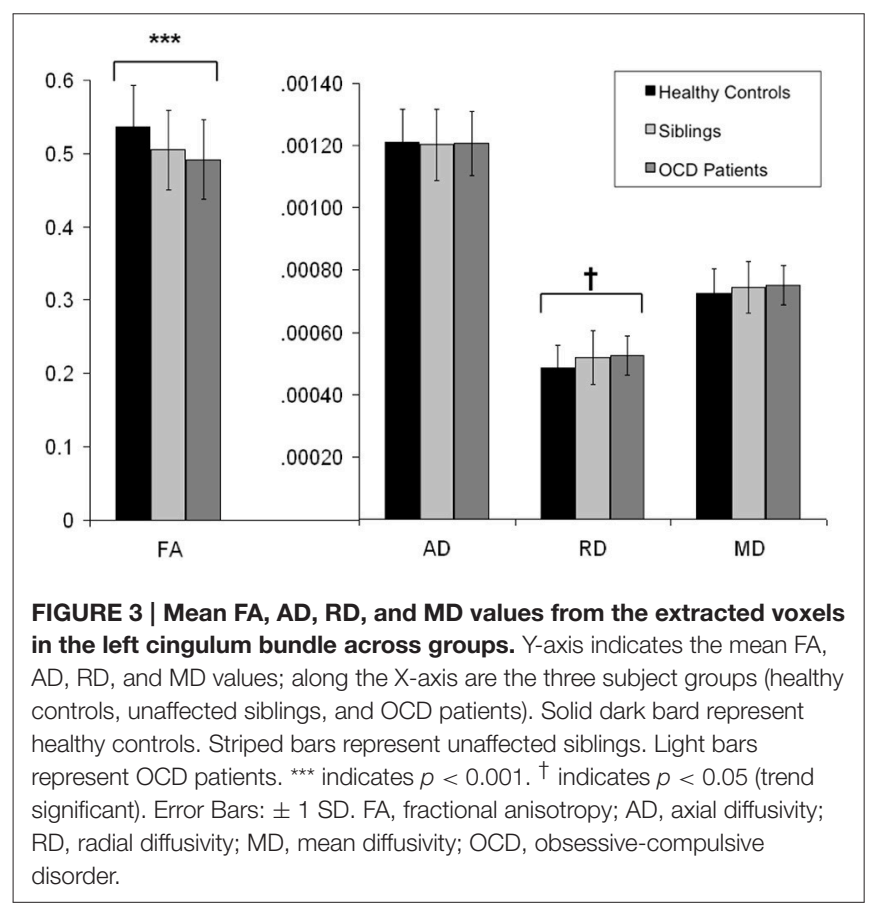

values did not differ across groups. Mean $\mathrm{RD}$ revealed a reverse pattern to that seen for FA: RD was trend-significantly higher in OCD patients compared to HC, with intermediate values in unaffected siblings. Such changes in diffusion parameters are in line with findings from previous DTI studies in OCD
TABLE 3 | Means and standard deviations of FA, AD, RD, and MD values for subject groups of healthy controls, unaffected siblings and OCD patients in the left cingulum bundle.

\begin{tabular}{|c|c|c|c|}
\hline Left cingulum bundle & $\begin{array}{c}\text { HC } \\
N=37 \\
\text { Mean } \pm \text { SD }\end{array}$ & $\begin{array}{c}\text { Siblings } \\
\begin{array}{c}N=15 \\
\text { Mean } \pm \text { SD }\end{array}\end{array}$ & $\begin{array}{l}\text { OCD patients } \\
\qquad \begin{array}{c}N=44 \\
\text { Mean } \pm \text { SD }\end{array}\end{array}$ \\
\hline FA & $0.54 \pm 0.06$ & $0.51 \pm 0.05$ & $0.49 \pm 0.05^{\star \star \star}$ \\
\hline$A D$ & $120.9 \pm 10.9$ & $120.2 \pm 11.4$ & $120.8 \pm 10.3$ \\
\hline $\mathrm{RD}$ & $48.5 \pm 7.3$ & $51.8 \pm 8.6$ & $52.5 \pm 6.4$ \\
\hline MD & $72.6 \pm 7.7$ & $74.6 \pm 8.3$ & $75.2 \pm 6.3$ \\
\hline
\end{tabular}

Diffusivities $A D, M D$, and $R D$ are given in units of $10^{-5} \mathrm{~mm}^{2} / \mathrm{s}$. ${ }^{\star \star *}$ indicates $p<.001$ compared with healthy controls. $A D$, axial diffusivity; $R D$, radial diffusivity; $M D$, mean diffusivity; $O C D$, obsessive-compulsive disorder; $H C$, healthy controls.

(Bora et al., 2011; Fan et al., 2012). Although Song et al. (2003) suggested a higher RD to be associated with demyelination, others have shown that the interpretation of changes in $\mathrm{RD}$ and $\mathrm{AD}$ needs careful consideration, due to the inherent disbalance between fiber thickness and imaging resolution (WheelerKingshott and Cercignani, 2009). Although a few genetic studies suggested myelination in the OCD pathophysiology (Zai et al., 2004; Stewart et al., 2007), we can only speculate about possible disruption of myelin integrity contributing to the white matter abnormalities in the left cingulum bundle. A similar pattern of lower FA in combination with higher $\mathrm{RD}$ (and normal $\mathrm{AD}$ values) has also been reported for autism, schizophrenia, and depression (Alexander et al., 2007; 
Ashtari et al., 2007; Lee et al., 2007; Michael et al., 2008; Seal et al., 2008; Whitford et al., 2010; Korgaonkar et al., 2011).

With regard to both FA and $\mathrm{RD}$, the unaffected siblings seem to represent an intermediate group between patients and $\mathrm{HC}$, suggesting that white matter alterations can be considered, at least partly, an endophenotype of OCD. The identification of disease endophenotypes can help to identify possible genetic risk factors of diseases and environmental effects. Menzies et al. (2008b) also found abnormal FA values in OCD patients as well as their first-degree relatives, although in that study abnormalities were mainly reported in the parietal and medial frontal regions.

To our knowledge, this is the first study investigating the white matter endophenotype of un-medicated OCD by exploring FA in combination with AD and RD. The sample sizes of the OCD and control groups were fairly large compared to previous studies and the ROIs were selected based on the most recent meta-analysis (Radua et al., 2014). An important limitation of the present study is that the reported findings were not corrected for multiple comparisons as we identified 7 ROIs a priori. In addition, we can't exclude the long-term effects of past medication use, although a 4-week washout period is thought as a sufficient time period for wearing off direct medication effects. Finally, only 15 unaffected siblings were included in the study. Due to the limited sample size, we can't rule out the possibility that the observation that the unaffected siblings were neither significantly different from OCD patients nor the HC might be due to the limited statistical power.

\section{REFERENCES}

Alexander, A. L., Lee, J. E., Lazar, M., Boudos, R., DuBray, M. B., Oakes, T. R., et al. (2007). Diffusion tensor imaging of the corpus callosum in Autism. Neuroimage 34, 61-73. doi: 10.1016/j.neuroimage.2006.08.032

Ashtari, M., Cottone, J., Ardekani, B. A., Cervellione, K., Szeszko, P. R., Wu, J., et al. (2007). Disruption of white matter integrity in the inferior longitudinal fasciculus in adolescents with schizophrenia as revealed by fiber tractography. Arch. Gen. Psychiatry 64, 1270-1280. doi: 10.1001/archpsyc.64.11.1270

Benedetti, F., Giacosa, C., Radaelli, D., Poletti, S., Pozzi, E., Dallaspezia, S., et al. (2013). Widespread changes of white matter microstructure in obsessivecompulsive disorder: effect of drug status. Eur. Neuropsychopharmacol. 23, 581-593. doi: 10.1016/j.euroneuro.2012.07.002

Bora, E., Harrison, B. J., Fornito, A., Cocchi, L., Pujol, J., Fontenelle, L. F., et al. (2011). White matter microstructure in patients with obsessive-compulsive disorder. J. Psychiatry Neurosci. 36, 42-46. doi: 10.1503/jpn.100082

Chamberlain, S. R., Menzies, L., Hampshire, A., Suckling, J., Fineberg, N. A., del Campo, N., et al. (2008). Orbitofrontal dysfunction in patients with obsessivecompulsive disorder and their unaffected relatives. Science 321, 421-422. doi: 10.1126/science. 1154433

Cohen, L. J., Hollander, E., DeCaria, C. M., Stein, D. J., Simeon, D., Liebowitz, M. R., et al. (1996). Specificity of neuropsychological impairment in obsessivecompulsive disorder: a comparison with social phobic and normal control subjects. J. Neuropsychiatry Clin. Neurosci. 8, 82-85. doi: 10.1176/jnp.8.1.82

de Vries, F. E., de Wit, S. J., Cath, D. C., van der Werf, Y. D., van der Borden, V., van Rossum, T. B., et al. (2014). Compensatory frontoparietal activity during working memory: an endophenotype of obsessive-compulsive disorder. Biol. Psychiatry 76, 878-887. doi: 10.1016/j.biopsych.2013.11.021

de Wit, S. J., Alonso, P., Schweren, L., Mataix-Cols, D., Lochner, C., Menchón, J. M., et al. (2014). Multicenter voxel-based morphometry mega-analysis of
In conclusion, this DTI study shows white matter alterations in OCD patients, un-medicated for at least 4 weeks, compared with $\mathrm{HC}$, mainly in the left cingulum bundle. A lower FA seems to be related to trend-wise higher $\mathrm{RD}$, suggesting potential disruption of myelin integrity in this region. The fact that the unaffected siblings represent an intermediate group between OCD patients and $\mathrm{HC}$ is suggestive for a white matter endophenotype of $\mathrm{OCD}$, reflecting genetic vulnerability.

\section{AUTHOR CONTRIBUTIONS}

SF contributed to data analysis and paper writing. $\mathrm{OH}$ contributed to daily supervision on the research analyses and paper writing. DC, YV, and DV contributed to supervision on the research. SD and FD contributed to data collection. PP contributed to technical supports and daily supervision on the research analyses and paper writing.

\section{ACKNOWLEDGMENTS}

This study was supported by FP7-People-2012-ITN grant (316978, project: TS-Eurotrain), the Dutch Organization for Scientific Research (NWO) with a ZonMW VENI grant (91686-038 to OV) and ZonMW AGIKO grant (920-03-542 to FD), a NARSAD young investigators award of the Brain \& Behavior Research Foundation (to OV), the Amsterdam Brain Imaging Platform, the Netherlands Brain Foundation (2010(1)-50 to OV), the Stichting tot Steun VCVGZ (STO957 to DC).

structural brain scans in obsessive-compulsive disorder. Am. J. Psychiatry 171, 340-349. doi: 10.1176/appi.ajp.2013.13040574

de Wit, S. J., de Vries, F. E., van der Werf, Y. D., Cath, D. C., Heslenfeld, D. J., Veltman, E. M., et al. (2012). Presupplementary motor area hyperactivity during response inhibition: a candidate endophenotype of obsessive-compulsive disorder. Am. J. Psychiatry 169, 1100-1108. doi: 10.1176/appi.ajp.2012.12010073

den Braber, A., van't Ent, D., Boomsma, D. I., Cath, D. C., Veltman, D. J., Thompson, P. M., et al. (2011). White matter differences in monozygotic twins discordant or concordant for obsessive-compulsive symptoms: a combined diffusion tensor imaging/voxel-based morphometry study. Biol. Psychiatry 70, 969-977. doi: 10.1016/j.biopsych.2011.03.029

Fan, Q., Yan, X., Wang, J., Chen, Y., Wang, X., Li, C., et al. (2012). Abnormalities of white matter microstructure in unmedicated obsessivecompulsive disorder and changes after medication. PLOS ONE 7:e35889. doi: 10.1371/journal.pone.0035889

First, M. B. S. R., Gibbon, M., and Williams, J. B. W. (2002). Structured Clinical Interview for DSM-IV-TR Axis I Disorder, Research Version . Patient Edition (SCID-I/P) ed. (New York, NY: Biometrics Research; New York State Psychiatric Institute).

Foa, E. B., Huppert, J. D., Leiberg, S., Langner, R., Kichic, R., Hajcak, G., et al. (2002). The Obsessive-Compulsive Inventory: development and validation of a short version. Psychol. Assess. 14, 485-496. doi: 10.1037/1040-3590.1 4.4.485

Goodman, W. K., Price, L. H., Rasmussen, S. A., Mazure, C., Delgado, P., Heninger, G. R., et al. (1989). The yale-brown obsessive compulsive scale. II. Validity. Arch Gen Psychiatry 46, 1012-1016. doi: 10.1001/archpsyc.1989.01810110054008

Gottesman, I. I., and Gould, T. D. (2003). The endophenotype concept in psychiatry: etymology and strategic intentions. Am. J. Psychiatry 160, 636-645. doi: 10.1176/appi.ajp.160.4.636 
Grisham, J. R., Anderson, T. M., and Sachdev, P. S. (2008). Genetic and environmental influences on obsessive-compulsive disorder. Eur. Arch. Psychiatry Clin. Neurosci. 258, 107-116. doi: 10.1007/s00406-00 7-0789-0

Hasan, K. M. (2006). Diffusion tensor eigenvalues or both mean diffusivity and fractional anisotropy are required in quantitative clinical diffusion tensor MR reports: fractional anisotropy alone is not sufficient. Radiology 239, 611-612; author reply 612-613. doi: 10.1148/radiol.2392051172

Hudziak, J. J., Van Beijsterveldt, C. E., Althoff, R. R., Stanger, C., Rettew, D. C., Nelson, E. C., et al. (2004). Genetic and environmental contributions to the Child Behavior Checklist Obsessive-Compulsive Scale: a cross-cultural twin study. Arch. Gen. Psychiatry 61, 608-616. doi: 10.1001/archpsyc.6 1.6.608

Jones, D. K., Christiansen, K. F., Chapman, R. J., and Aggleton, J. P. (2012). Distinct subdivisions of the cingulum bundle revealed by diffusion MRI fibre tracking: implications for neuropsychological investigations. Neuropsychologia 51, 67-78. doi: 10.1016/j.neuropsychologia.2012.11.018

Koch, K., Reess, T. J., Rus, O. G., Zimmer, C., and Zaudig, M. (2014). Diffusion tensor imaging (DTI) studies in patients with obsessivecompulsive disorder (OCD): a review. J. Psychiatr. Res. 54, 26-35. doi: 10.1016/j.jpsychires.2014.03.006

Korgaonkar, M. S., Grieve, S. M., Koslow, S. H., Gabrieli, J. D., Gordon, E., and Williams, L. M. (2011). Loss of white matter integrity in major depressive disorder: evidence using tract-based spatial statistical analysis of diffusion tensor imaging. Hum. Brain Mapp. 32, 2161-2171. doi: 10.1002/hbm. 21178

Lázaro, L., Bargalló, N., Castro-Fornieles, J., Falcón, C., Andrés, S., Calvo, R., et al. (2009). Brain changes in children and adolescents with obsessive-compulsive disorder before and after treatment: a voxel-based morphometric MRI study. Psychiatry Res. 172, 140-146. doi: 10.1016/j.pscychresns.2008.12.007

Lázaro, L., Castro-Fornieles, J., Cullell, C., Andrés, S., Falcón, C., Calvo, R., et al. (2011). A voxel-based morphometric MRI study of stabilized obsessivecompulsive adolescent patients. Prog. Neuropsychopharmacol. Biol. Psychiatry 35, 1863-1869. doi: 10.1016/j.pnpbp.2011.07.016

Le Bihan, D., Mangin, J. F., Poupon, C., Clark, C. A., Pappata, S., Molko, N., et al. (2001). Diffusion tensor imaging: concepts and applications. J. Magn. Reson. Imaging 13, 534-546. doi: 10.1002/jmri.1076

Lee, J. E., Bigler, E. D., Alexander, A. L., Lazar, M., DuBray, M. B., Chung, M. K., et al. (2007). Diffusion tensor imaging of white matter in the superior temporal gyrus and temporal stem in autism. Neurosci. Lett. 424, 127-132. doi: 10.1016/j.neulet.2007.07.042

Menzies, L., Chamberlain, S. R., Laird, A. R., Thelen, S. M., Sahakian, B. J., and Bullmore, E. T. (2008a). Integrating evidence from neuroimaging and neuropsychological studies of obsessive-compulsive disorder: the orbitofronto-striatal model revisited. Neurosci. Biobehav. Rev. 32, 525-549. doi: 10.1016/j.neubiorev.2007.09.005

Menzies, L., Williams, G. B., Chamberlain, S. R., Ooi, C., Fineberg, N., Suckling, J., et al. (2008b). White matter abnormalities in patients with obsessivecompulsive disorder and their first-degree relatives. Am. J. Psychiatry 165, 1308-1315. doi: 10.1176/appi.ajp.2008.07101677

Michael, A. M., Calhoun, V. D., Pearlson, G. D., Baum, S. A., and Caprihan, A. (2008). Correlations of diffusion tensor imaging values and symptom scores in patients with schizophrenia. Conf. Proc. IEEE Eng. Med. Biol. Soc. 2008, 5494-5497. doi: 10.1109/iembs.2008.4650458

Montgomery, S. A., and Asberg, M. (1979). A new depression scale designed to be sensitive to change. Br. J. Psychiatry 134, 382-389. doi: 10.1192/bjp.13 4.4.382

Nestadt, G., Grados, M., and Samuels, J. F. (2000). Genetics of obsessivecompulsive disorder. Psychiatr. Clin. North Am. 33, 141-158. doi: 10.1016/j.psc.2009.11.001

Oldfield, R. C. (1971). The assessment and analysis of handedness: the Edinburgh inventory. Neuropsychologia 9, 97-113. doi: 10.1016/0028-3932(71)90067-4

Peters, B. D., Szeszko, P. R., Radua, J., Ikuta, T., Gruner, P., DeRosse, P., et al. (2012). White matter development in adolescence: diffusion tensor imaging and meta-analytic results. Schizophr. Bull. 38, 1308-1317. doi: $10.1093 / \mathrm{schbul} / \mathrm{sbs} 054$

Piras, F., Piras, F., Chiapponi, C., Girardi, P., Caltagirone, C., and Spalletta, G. (2013). Widespread structural brain changes in OCD: a systematic review of voxel-based morphometry studies. Cortex 62, 89-108. doi: 10.1016/j.cortex.2013.01.016

Radua, J., Grau, M., van den Heuvel, O. A., Thiebaut de Schotten, M., Stein, D. J., Canales-Rodríguez, E. J., et al. (2014). Multimodal voxel-based metaanalysis of white matter abnormalities in obsessive-compulsive disorder. Neuropsychopharmacology 39, 1547-1557. doi: 10.1038/npp.2014.5

Radua, J., and Mataix-Cols, D. (2009). Voxel-wise meta-analysis of grey matter changes in obsessive-compulsive disorder. Br. J. Psychiatry 195, 393-402. doi: 10.1192/bjp.bp.108.055046

Riffkin, J., Yücel, M., Maruff, P., Wood, S. J., Soulsby, B., Olver, J., et al. (2005). A manual and automated MRI study of anterior cingulate and orbito-frontal cortices, and caudate nucleus in obsessive-compulsive disorder: comparison with healthy controls and patients with schizophrenia. Psychiatry Res. 138, 99-113. doi: 10.1016/j.pscychresns.2004.11.007

Rotge, J. Y., Guehl, D., Dilharreguy, B., Tignol, J., Bioulac, B., Allard, M., et al. (2009). Meta-analysis of brain volume changes in obsessive-compulsive disorder. Biol. Psychiatry 65, 75-83. doi: 10.1016/j.biopsych.2008.06.019

Savage, C. R., Baer, L., Keuthen, N. J., Brown, H. D., Rauch, S. L., and Jenike, M. A. (1999). Organizational strategies mediate nonverbal memory impairment in obsessive-compulsive disorder. Biol. Psychiatry 45, 905-916. doi: 10.1016/S0006-3223(98)00278-9

Schmahmann, J., and Pandya, D. (2006). Fiber Pathways of the Brain. New York, NY: Oxford University Press.

Schuurmans, J., van Balkom, A. J., van Megen, H. J., Smit, J. H., Eikelenboom, M., Cath, D. C., et al. (2012). The Netherlands Obsessive Compulsive Disorder Association (NOCDA) study: design and rationale of a longitudinal naturalistic study of the course of OCD and clinical characteristics of the sample at baseline. Int. J. Methods Psychiatr. Res. 21, 273-285. doi: 10.1002/mpr.1372

Seal, M. L., Yücel, M., Fornito, A., Wood, S. J., Harrison, B. J., Walterfang, M., et al. (2008). Abnormal white matter microstructure in schizophrenia: a voxelwise analysis of axial and radial diffusivity. Schizophr. Res. 101, 106-110. doi: 10.1016/j.schres.2007.12.489

Shaw, P., Sharp, W., Sudre, G., Wharton, A., Greenstein, D., Raznahan, A., et al. (2015). Subcortical and cortical morphological anomalies as an endophenotype in obsessive-compulsive disorder. Mol. Psychiatry 20, 224-231. doi: $10.1038 / \mathrm{mp} .2014 .3$

Smith, S. M., and Nichols, T. E. (2009). Threshold-free cluster enhancement: addressing problems of smoothing, threshold dependence and localisation in cluster inference. Neuroimage 44, 83-98. doi: 10.1016/j.neuroimage.2008.03.061

Song, S. K., Sun, S. W., Ju, W. K., Lin, S. J., Cross, A. H., and Neufeld, A. H. (2003). Diffusion tensor imaging detects and differentiates axon and myelin degeneration in mouse optic nerve after retinal ischemia. Neuroimage 20, 1714-1722. doi: 10.1016/j.neuroimage.2003.07.005

Stewart, S. E., Platko, J., Fagerness, J., Birns, J., Jenike, E., Smoller, J. W., et al. (2007). A genetic family-based association study of OLIG2 in obsessive-compulsive disorder. Arch. Gen. Psychiatry 64, 209-214. doi: 10.1001/archpsyc.64.2.209

Szczepankiewicz, F., Lasic, S., van Westen, D., Sundgren, P. C., Englund, E., Westin, C. F., et al. (2014). Quantification of microscopic diffusion anisotropy disentangles effects of orientation dispersion from microstructure: applications in healthy volunteers and in brain tumors. Neuroimage 104, 241-252. doi: 10.1016/j.neuroimage.2014.09.057

Togao, O., Yoshiura, T., Nakao, T., Nabeyama, M., Sanematsu, H., Nakagawa, A., et al. (2010). Regional gray and white matter volume abnormalities in obsessivecompulsive disorder: a voxel-based morphometry study. Psychiatry Res. 184, 29-37. doi: 10.1016/j.pscychresns.2010.06.011

van den Heuvel, O. A., Remijnse, P. L., Mataix-Cols, D., Vrenken, H., Groenewegen, H. J., Uylings, H. B., et al. (2009). The major symptom dimensions of obsessive-compulsive disorder are mediated by partially distinct neural systems. Brain 132, 853-868. doi: 10.1093/brain/ awn267

Van Grootheest, D. S., Cath, D. C., Beekman, A. T., and Boomsma, D. I. (2007) Genetic and environmental influences on obsessive-compulsive symptoms in adults: a population-based twin-family study. Psychol. Med. 37, 1635-1644. doi: $10.1017 /$ s0033291707000980

Wheeler-Kingshott, C. A., and Cercignani, M. (2009). About "axial" and "radial" diffusivities. Magn. Reson. Med. 61, 1255-1260. doi: 10.1002/mrm.21965 
Whitford, T. J., Kubicki, M., Schneiderman, J. S., O’Donnell, L. J., King, R., Alvarado, J. L., et al. (2010). Corpus callosum abnormalities and their association with psychotic symptoms in patients with schizophrenia. Biol. Psychiatry 68, 70-77. doi: 10.1016/j.biopsych.2010. 03.025

Yoo, S. Y., Jang, J. H., Shin, Y. W., Kim, D. J., Park, H. J., Moon, W. J., et al. (2007). White matter abnormalities in drug-naive patients with obsessivecompulsive disorder: a diffusion tensor study before and after citalopram treatment. Acta Psychiatr. Scand. 116, 211-219. doi: 10.1111/j.1600-0447.2007. 01046.x

Zai, G., Bezchlibnyk, Y. B., Richter, M. A., Arnold, P., Burroughs, E., Barr, C. L., et al. (2004). Myelin oligodendrocyte glycoprotein (MOG) gene is associated with obsessive-compulsive disorder. Am. J. Med. Genet. B Neuropsychiatr. Genet. 129B, 64-68. doi: 10.1002/ajmg.b. 30077
Conflict of Interest Statement: The authors declare that the research was conducted in the absence of any commercial or financial relationships that could be construed as a potential conflict of interest.

The Associate Editor Kirsten R Müller-Vahl declares that, despite of having collaborated with Danielle C. Cath in the EU funded MC-ITN TS-Eurotrain, the review process was handled objectively.

Copyright (๑ 2016 Fan, van den Heuvel, Cath, van der Werf, de Wit, de Vries, Veltman and Pouwels. This is an open-access article distributed under the terms of the Creative Commons Attribution License (CC BY). The use, distribution or reproduction in other forums is permitted, provided the original author(s) or licensor are credited and that the original publication in this journal is cited, in accordance with accepted academic practice. No use, distribution or reproduction is permitted which does not comply with these terms. 